\title{
La samba à Rio de Janeiro et le paradigme de l'Estácio
}

The Samba in Rio de Janeiro and the Estacio Paradigm

\section{Carlos Sandroni}

\section{(2) OpenEdition}

Journals

Édition électronique

URL : http://journals.openedition.org/ethnomusicologie/857

ISSN : 2235-7688

Éditeur

ADEM - Ateliers d'ethnomusicologie

Édition imprimée

Date de publication : 1 décembre 1997

Pagination : 153-168

ISBN : 2-8257-0579-9

ISSN : 1662-372X

\section{Référence électronique}

Carlos Sandroni, « La samba à Rio de Janeiro et le paradigme de l'Estácio », Cahiers d'ethnomusicologie [En ligne], 10 | 1997, mis en ligne le 06 janvier 2012, consulté le 19 avril 2019. URL : http:// journals.openedition.org/ethnomusicologie/857

Ce document a été généré automatiquement le 19 avril 2019.

Tous droits réservés 


\section{La samba ${ }^{1}$ à Rio de Janeiro et le paradigme de l'Estácio}

The Samba in Rio de Janeiro and the Estacio Paradigm

\section{Carlos Sandroni}

\section{Les deux sambas}

1 Les chercheurs qui se sont occupés de l'histoire de la samba à Rio de Janeiro y ont remarqué l'existence de deux styles successifs. Le premier correspondrait à la période qui va de 1917 (l'année du succès de Pelo telefone, jalon initial du genre²) jusqu'à la fin des années 1920 environ; le second se serait établi vers le début des annés 1930, le moment donc où la samba contemporaine aurait trouvé, à quelques retouches près, sa version définitive.

2 Les principaux sambistas associés à la samba « ancien style » sont Sinhô (José Batista da Silva, 1888-1930), connu dans les années 1920 comme «le Roi de la samba», Donga (Ernesto dos Santos, 1889-1974), l'auteur de Pelo telefone, et João da Baiana (João Machado Guedes, 1887-1974). Les deux derniers étaient des fils de Bahianaises, et tous les trois furent, dans leur jeunesse, des habitués des fêtes musicales organisées par une autre vieille Bahianaise éminente, Tia ( tante ») Ciata. C'est pourquoi le style de leurs sambas fut associé à la maison de celle-ci et à son quartier, la Cidade Nova.

Les sambistas identifiés à la samba "nouveau style» étaient en revanche presque tous originaires du quartier de l'Estácio de Sá à Rio. Le plus connu d'entre eux s'appelait Ismael Silva (1905-1978) et, parmi les autres, on peut mentionner Bide (Alcebíades Barcelos, 1902-1975), Nilton Bastos (1899-1931) et Brancura (Sílvio Fernandes, 1908-1935). 
Le compositeur Ismael Silva et un groupe de chanteuses.

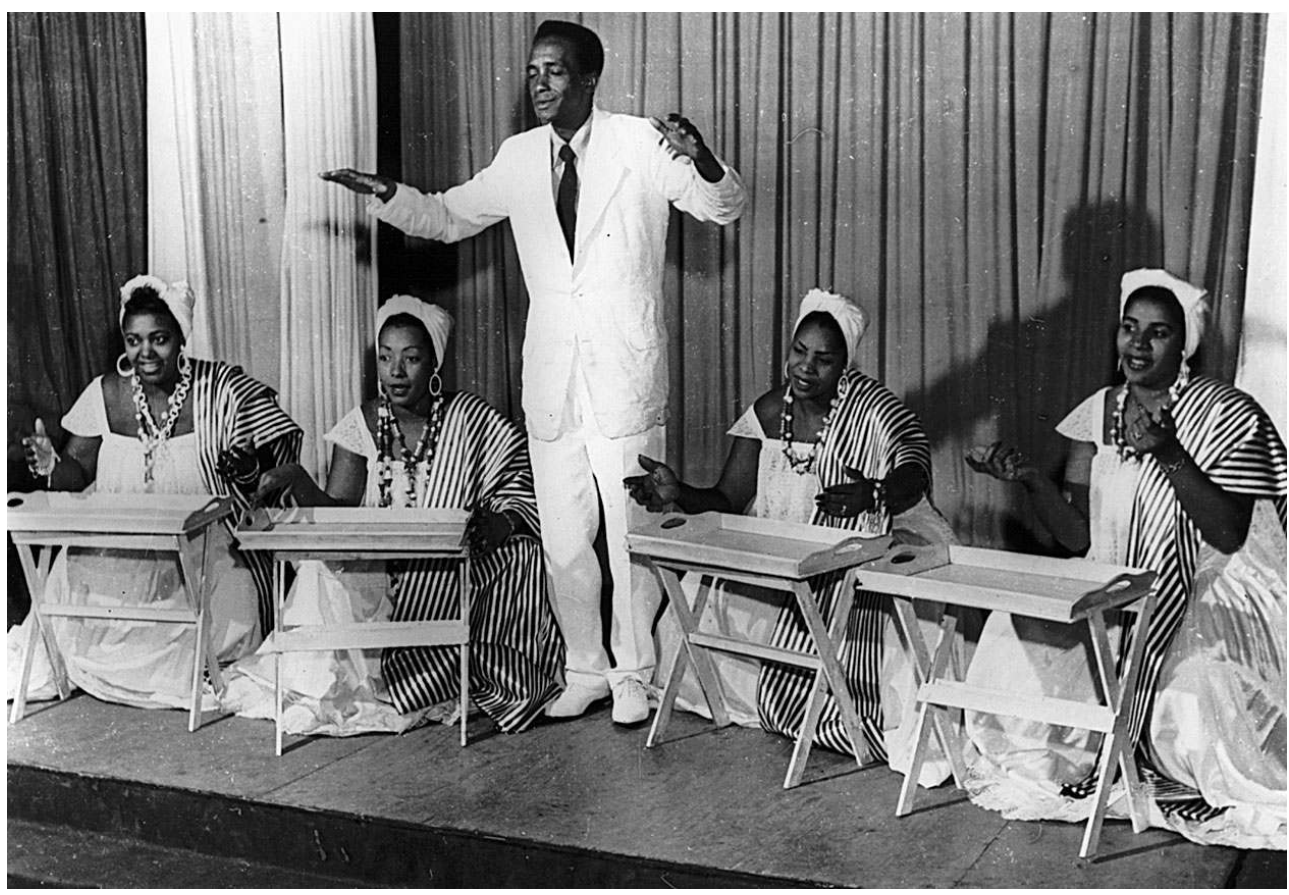

Dans la littérature spécialisée, il n’y a aucune description détaillée des caractéristiques musicales des deux styles ; pour les Brésiliens, la différence se reconnaît «à l'oreille». Comme l'écrit le journaliste Sérgio Cabral : «C'est facile : il suffit de comparer un vieil enregistrement d'une samba de Sinhô (ou même Pelo telefone) avec un autre de n'importe quelle samba composée par les musiciens de l'Estácio de Sá pour établir la différence entre les deux formes de samba » (Cabral $1974: 21$ ). « Il suffit de comparer », c'est-à-dire qu'il suffit d'entendre un enregistrement après l'autre : la différence saute aux yeux (ou aux oreilles), sans qu'aucun commentaire verbal soit nécessaire.

5 Le témoignage peut-être le plus important à ce sujet a été recueilli par le même Cabral qui, dans un entretien, confronta deux compositeurs considérés comme représentatifs respectivement du style en vigueur jusqu'à la fin des années 1920 et de celui qui s'imposa à partir des années 1930: Donga et Ismael Silva. Cabral leur posa la même question: «qu'est-ce que la samba?» Donga répondit avec l'exemple de Pelo telefone et Ismael répliqua : "Ceci est un maxixe ». Pour lui, Se você jurar (composée par lui-même et Nilton Bastos en 1931) serait une véritable samba. Mais Donga divergea aussi : «Ceci n'est pas une samba, c'est une marche » (Cabral $1974: 21-2)^{3}$.

6 La plupart des critiques donnèrent raison à Ismael Silva. Si nous ne connaissons aucun commentateur qui mette en cause l'appartenance de Se vocêjurar au genre samba, ce n'est pas le cas avec Pelo telefone. Máximo et Didier n'hésitent pas à l'appeler samba maxixée (1990 : 118). Pour Silva et Oliveira Filho, aussi, les sambas en style ancien, plutôt que maxixées, seraient tout simplement des maxixes. Le responsable de cette confusion serait Donga, qui aurait abusivement qualifié Pelo telefone de samba. En raison du succès obtenu par cette composition, « le mot samba, qui jusqu'alors était utilisé en synonymie presque parfaite avec tango et maxixe, a vu son sens se préciser, en se substituant dans la pratique aux deux autres. Les sambas de Sinhô sont donc en réalité des maxixes « (Silva et Oliveira Filho 1989: 45). Alvarenga pense la même chose car, pour elle, "non seulement Pelo 
telefone, mais tous les autres morceaux de Sinhô, le premier grand créateur de Sambas, ne se distinguent pas réellement du Maxixe chanté » (Alvarenga 1982 : 343).

Or le maxixe est un genre de musique à danser créé à Rio vers 1880. L'accompagnement de la samba ancien style était, comme celui des vieux maxixes, basé sur des figures rythmiques très communes dans la musique de danse sud-américaine du XIX ${ }^{\mathrm{e}}$ siècle. Il s'agit notamment de ce que le grand musicologue brésilien Mário de Andrade a appelé « syncope caractéristique » et que les Cubains ont appelé tresillo:

Ex.1
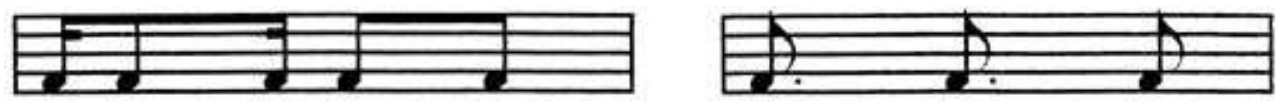

8 A partir de 1930, l'accompagnement des sambas a abandonné de telles formules rythmiques. Elles ont été remplacées par la batucada, c'est-à-dire la polyrythmie assurée par un ensemble d'instruments, dont le surdo, la cuíca et le tamborim.

$9 \quad$ Le tamborim est un petit tambour sur cadre frappé à l'aide d'une baguette. Le son qu'il produit est bref, sec et précis; il se distingue dans la samba par une attaque très nette, ce qui facilite sa notation rythmique par les moyens conventionnels. Cet instrument étant associé à un groupe de figures rythmiques particulièrement importantes pour la définition du nouveau style, nous avons créé le concept du " paradigme de l'Estácio » afin de cerner les propriétés formelles de ce groupe.

\section{Le paradigme de l'Estácio}

10 Les rythmes constituant le paradigme de l'Estácio ont déjà été décrits par quelques auteurs. L'ethnomusicologue zaïrois Kazadi-wa Mukuna se réfère, dans son ouvrage sur les éléments bantous dans la musique populaire brésilienne, à un certain «cycle rythmique » (auquel il ne donne pas de nom particulier) qui serait présent dans la samba et qui « n'a pas été discuté par les chercheurs » (Mukuna s.d. : 82-83) :

Ex. 2

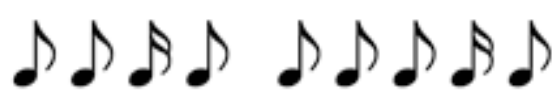

11 Ce cycle relativement nouveau est opposé selon lui à la "syncope caractéristique ", qui serait aussi présente dans la samba, mais en tant qu'héritage du lundu $u^{5}(\mathrm{idem}: 80-81$ ). Mukuna donne une variation de ce rythme :

Ex. 3

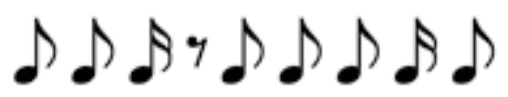

Aussi bien la "syncope caractéristique » que ce cycle et sa variante se retrouvent, selon Mukuna, dans la musique de quelques régions du Zaïre.

13 Mukuna poursuit en affirmant que, « de ces deux rythmes de samba, à savoir, celui hérité du lundu et le [nouveau] cycle, ce dernier peut être considéré comme le rythme de samba le plus représentatif » (ibid. : 82). Et plus loin il parle de « Rio de Janeiro, où la division du 
cycle rythmique en seize pulsations [à savoir, la figure rythmique en question] serait introduite dans la samba, caractérisant ainsi la samba 'carioca', c'est-à-dire 'de Rio de Janeiro" (ibid.: 205). Or la «syncope caractéristique» était la formule d'accompagnement préférée de la samba ancien style. Si les auteurs cités plus haut assimilaient ce dernier au maxixe, Mukuna préfère l'associer au lundu, ce qui n'est pas étonnant car l'historiographie de la musique brésilienne a très souvent fait dériver le premier du second. En outre, si Mukuna considère le « nouveau » cycle comme étant à la fois le plus représentatif et celui de la samba carioca, c'est bien parce qu'il voit son association au style dominant à Rio depuis les années 30 .

Enfin, Mukuna affirme que le nouveau cycle « est fréquemment donné par le tamborim dans l'orchestration de la percussion » (ibid. : 82). L'association au tamborim est renforcée par Araújo, qui donne un exemple similaire à celui de Mukuna et appelle celui-ci tamborim cycle ou tamborim pattern (Araújo 1992 : 146-147) :

Ex. 4

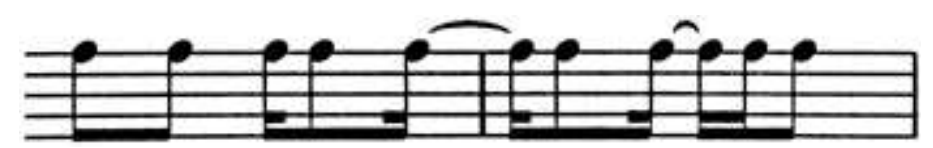

Kubik, quant à lui, écrit : «Quiconque d'un peu familiarisé avec la samba brésilienne de rue, telle qu'elle peut être vue à Rio de Janeiro à l'époque du carnaval [...] doit connaître une formule rythmique très caractéristique qui en est un des traits les plus constants. Elle peut être jouée sur différents instruments, par exemple sur un tambour aigu [c'est-à-dire le tamborim] [...] ou même sur une guitare. Il s'agit d'un élément central, dans lequel tous les autres musiciens, chanteurs et danseurs trouvent un point pivot pour leur orientation » (Kubik 1979 : 13) ${ }^{6}$.

Plus loin il transcrit, selon sa propre méthode, deux versions de cette figure rythmique, qu'il affirme par ailleurs être caractéristique aussi de certaines régions de l'Angola et du Zaïre :

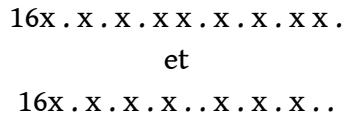

17 Le chiffre au début de la ligne indique le fait que le cycle complet présente seize pulsations. Kubik note par des « $\mathrm{x}$ » les pulsations où se trouvent des frappes, et par des points celles où il n'y en a pas. A titre de comparaison, ces deux exemples peuvent être transcrits en notation conventionnelle ${ }^{7}$ :

Ex. 5

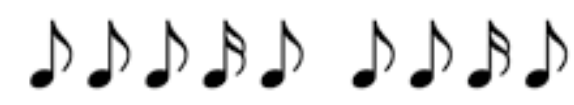

Ex. 6

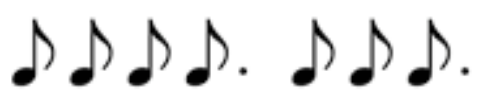

Kubik n'hésite pas à considérer comme équivalentes les deux figures ci-dessous, tout comme, à sa manière, l'avait fait Mukuna plus haut : 
Ex. 7

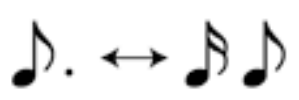

croches) et des valeurs ternaires (représentées par les croches pointées). Celui qui tient le surdo ne joue en revanche que des valeurs de type binaire (les noires). Il s'ensuit que chaque valeur ternaire jouée au tamborim produit un décalage par rapport au surdo. Si les premières croches du tamborim sont jouées - pour employer l'expression de Kolinski (1960 et 1973) - de façon cométrique, celles qui succèdent à la première croche pointée seront contramétriques, celles qui suivent la deuxième croche pointée seront à nouveau cométriques et ainsi de suite. (C'est pourquoi nous avons parlé de polyrythmie à propos de la batucada: il n'est pas possible de déduire, par division, le rythme du tamborim de celui du surdo.) 
Ex. 9

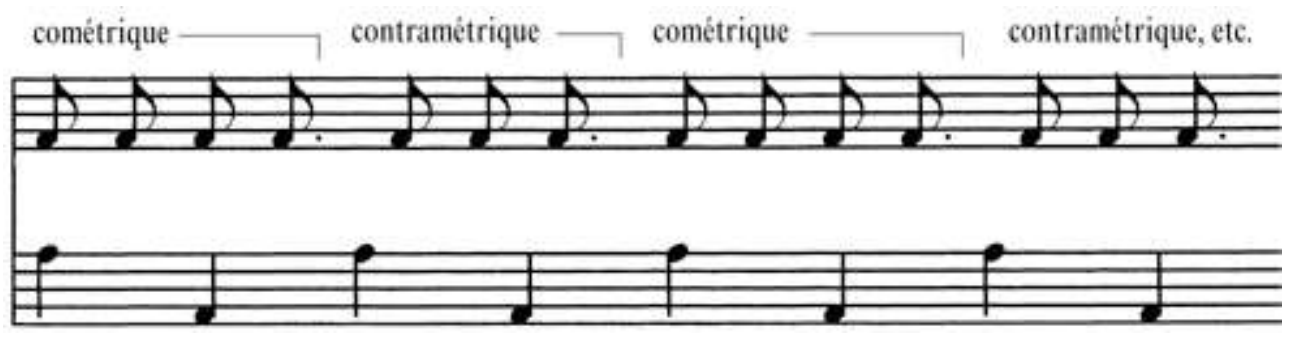

lensemble tamborim / surdo repose la question de la circularité à un autre niveau. En effet, les deux parties inégales du rythme de tamborim possèdent toujours, l'une, un début cométrique, et l'autre un début contramétrique. Mukuna, Araújo et Kubik ont considéré qu'il fallait mettre le côté cométrique au début; du moins ont-ils écrit ce rythme d'une façon qui rend cela implicite. Ce choix correspond parfaitement au penchant cométrisant de l'oreille et de l'écriture occidentales, mais pas forcément à la réalité.

\section{Ex. 10. Onde a dor ñao tem razão}
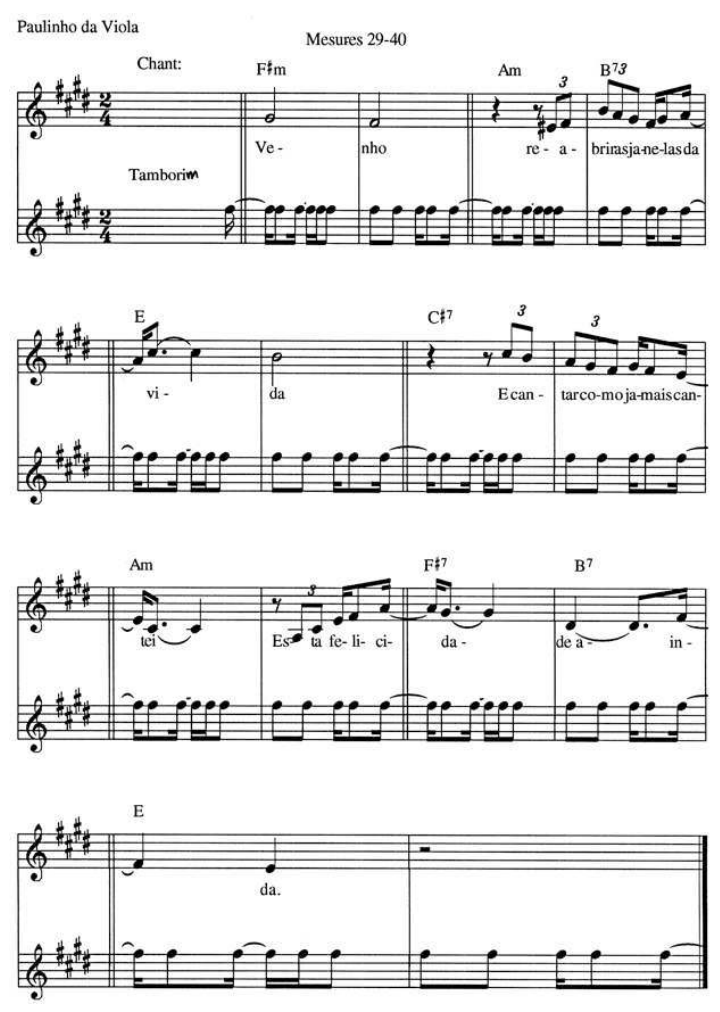

28 Il se pourrait bien que, pour les musiciens concernés, la façon de caler l'ensemble tamborim / surdo par rapport aux autres éléments de la samba ne soit pas arbitraire. Parmi 
ces éléments, il y a en particulier le rythme harmonique de l'accompagnement des guitares et cavaquinhos et les paroles des sambas, dont il arrive souvent que le rapport au temps ne soit pas circulaire, mais linéaire.

Dans l'exemple d'une samba de Paulinho da Viola, Onde a dor não tem razão $0^{8}$, les syllabes toniques sur lesquelles la phrase musicale s'appuie ( Venho/Reabrir as janelas da vida/E cantar como jamais cantei/Esta felicidade ainda »), comme les changements harmoniques principaux, arrivent toujours dans les mesures où le début est contramétrique. Ce modèle se répète invariablement dans toutes les sambas postérieures à 1935 environ, où la figure rythmique en question apparaît.

D'autre part, dans les enregistrements de samba où tous les instruments de la batucada attaquent dès la première mesure, le contraste entre tamborim et surdo commence seulement à partir de la fin de la deuxième mesure, comme cela se voit dans les quatre premières mesures de la même samba, Onde a dor não tem razão :

Ex. 11

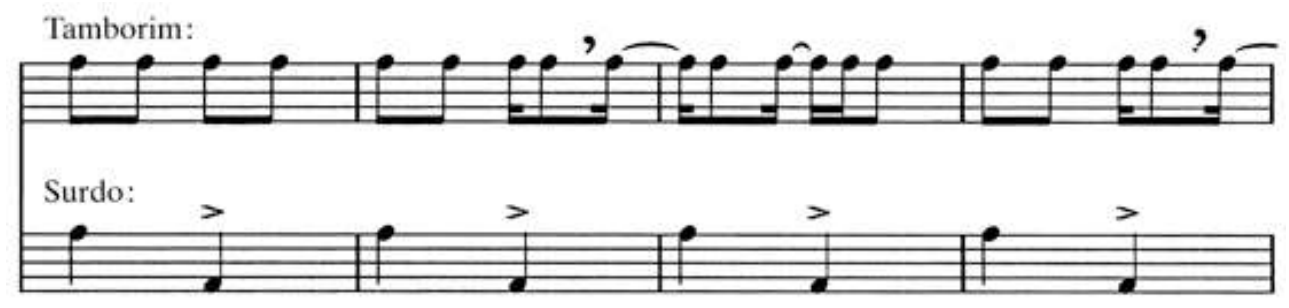

31 Dans le premier cycle de deux mesures de cet enregistrement, les deux mesures du tamborim possèdent un début cométrique. Dans le deuxième cycle de deux mesures - et du reste dans tous les cycles de deux mesures qui suivent - la première mesure possède en revanche un début contramétrique, et la deuxième un début cométrique. Cet exemple n'est en aucune manière exceptionnel : au contraire, il représente la règle au début d'une samba, en s'appliquant non seulement au cas du tamborim, mais aussi à tous les instruments de la samba qui se caractérisent par l'emploi de rythmes du même type, comme le cavaquinho et la guitare.

Cela semble indiquer que, le début de la batucada étant un point particulièrement sensible du point de vue de la coordination rythmique, le contraste entre les rythmes du tamborim et du surdo serait dans ce cas particulièrement perturbateur pour les musiciens. Si, cependant, le véritable point initial de la figure rythmique du tamborim était cométrique, comme le laissent supposer les exemples de Mukuna, Kubik et Araújo, la différence entre le premier cycle de deux mesures et tous les cycles suivants n'existerait pas car, dans ce cas, le même début cométrique serait répété tout au long du morceau. En vérité, la version qui présente un début cométrique est une formule de démarrage.

Une autre preuve de cela est que, quand le surdo démarre tout seul et que la figure du tamborim vient par la suite s'installer sur un fond métrique déjà clairement établi, elle commence de manière "normale ", à savoir, en l'ocurrence, de manière contramétrique. Cela peut s'entendre, par exemple, dans l'extrait du programme de radio « Aquarelas do Brasil », réalisé par Almirante à la Radio Nationale (Rio de Janeiro) le 4 mai 1945, ayant pour thème les Ecoles de Samba'. À un moment du programme, les instruments de la batucada sont présentés un à un: dans l'ordre surdo, tamborim, pandeiro et cuíca. Le tamborim démarre après le surdo, et il démarre de manière contramétrique : 


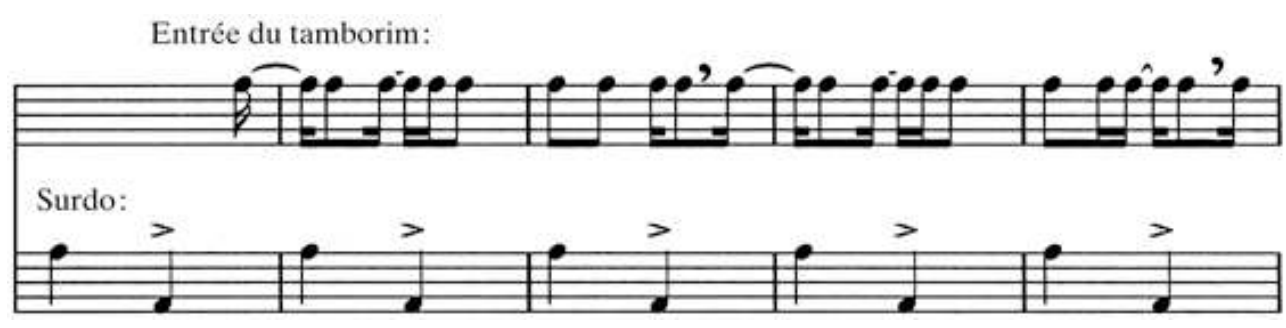

Encore une fois, il ne s'agit pas d'un exemple isolé, mais d'une façon de faire très courante dans les performances de samba à Rio de Janeiro.

\section{L'imparité rythmique}

Comment définir ce type de formule rythmique trouvée dans la samba carioca? Il faut tout d'abord remarquer que des formules de ce type ont été trouvées à maintes reprises dans la musique africaine. Pour s'y référer, Jones (1959: 8) a utilisé l'expression « rythmes additifs ", adoptée également par Nketia (1975: 131). Arom a fait la critique de cette expression ; pour la remplacer, il a proposé celle d'« imparité rythmique » (1985:429-31), qui est définie dans son ouvrage par la formule

$$
2 n=(n+1)+(n-1)
$$

où « $\mathrm{n}$ » est un chiffre pair égal ou plus grand que 4. Dans le cas présent, le cycle rythmique complet (2n) comporte seize doubles croches, d'où s'ensuit que

$$
16=(8+1)+(8-1) \text {. }
$$

Il s'agit donc d'une façon d'organiser le rythme où le cycle rythmique, bien que pair, est divisé en deux moitiés inégales, impaires. À la place de 8+8,9+7. Le 9 et le 7 , à leur tour, sont subdivisés en groupes de 2 et de 3 - les croches et doubles-croches, si l'on veut, de notre rythme de tamborim.

Cette formule est cependant insuffisante pour arriver à une distribution de croches et doubles-croches comme celle du rythme du tamborim. En effet, elle ne tient pas compte de l'agencement des groupes de 2 et 3 à l'intérieur de chaque partie. En appliquant la seule formule d'Arom, on pourrait arriver à des rythmes comme: $9+7=(3+3+3)+(3+2+2)$, qui n'ont rien à voir avec la samba carioca.

Pour être applicable ici, la formule doit, à un premier niveau, remplir une condition : la quantité de groupes de 3 doit toujours être égale à 2 . La quantité de groupes de 2 sera, par conséquent, égale à $\mathrm{n}-3$.

Si 2 n est égal à 16 , on obtient donc deux groupes de 3 et cinq groupes de 2, ce qui donne trois types de formules, selon la façon dont les groupes de 3 sont interpolés au milieu des groupes de 2 :

a) 33222223322222 ...

b) 32322223232222 ...

c) $32232223223222 \ldots$

Ces formules sont envisagées pour l'instant comme circulaires : elles n'ont pas encore un point d'entrée défini. Le 3 du début ne doit donc pas être conçu comme un vrai début, mais comme le produit d'un découpage arbitraire, imposé par l'écriture linéaire. 
41 carioca? En fait, la formule "a» n'y rentre pas: elle n'est jamais employée en ostinato dans les batucadas de samba. La formule $« b$ » est présente dans certains enregistrements de samba "nouveau style» du début des années 1930, mais elle n'est plus pratiquée aujourd'hui. Quant à la formule « c », finalement, elle est employée aujourd'hui dans la plupart des ostinati de tamborim, cavaquinho et guitare de la samba à Rio de Janeiro. La formule $2 \mathrm{n}=(\mathrm{n}+1)+(\mathrm{n}-1)$ peut donc être remplacée dans le cas de la samba carioca , avec plus de précision, par

$$
2 n=3+(n-2)+3+(n-4)
$$

Il s'agit, bien entendu, d'une formule qui ne s'applique pas seulement à la samba carioca, mais à beaucoup d'autres rythmes afro-américains et africains, comme le 3-3-2, qu'on rencontre dans la capoeira bahianaise et en Afrique par exemple dans la danse sovu (Jones 1959 : 114), et le 3-2-3-2-2 trouvé dans le candomblé bahianais et partout en Afrique (Jones $1959: 213)^{10}$.

Mais pour comprendre exactement ce que font les joueurs de tamborim dans la samba carioca, il faut aller un peu plus loin. La formule «c » était envisagée plus haut comme circulaire. $\mathrm{Si}$, par contre, elle est intégrée à une performance réelle de samba, un point d'entrée par rapport à l'ensemble doit être choisi. Il y a donc, dans une première approche, sept possibilités, puisque le cycle complet comporte autant d'articulations :
1) 2223223
2) 2232232
3) 2322322
4) 3223222
5) 2232223
6) 2322232
7) 3222322

Chacun des rythmes considérés peut cependant être calé de façon cométrique ou contramétrique. Les cinq groupes qui commencent par 2 donnent ainsi lieu à dix possibilités, cinq cométriques et cinq contramétriques. Les deux groupes qui commencent par 3 donnent lieu à six possibilités, deux cométriques et quatre contramétriques.

Or, parmi toutes ces possibilités, les musiciens de samba à Rio ne choisissent que les cinq qui commencent par 2 de façon contramétrique. En effet, dans la deuxième partie de cet article, les variantes 1 et 5 , qui commencent par 2, ont déjà été citées : la première choisie par Kubik, et la deuxième par Mukuna et Araújo. Par ailleurs, il a été montré que leur début devait être calé par rapport à l'ensemble de façon contramétrique, ce que les auteurs cités n'avaient pas précisé.

Il est en outre clair que ces deux variantes font partie d'un groupe formel qui en comporte trois autres, les cinq étant employées dans les performances de samba par les joueurs de tamborim, de cuíca, de cavaquinho (voir les exemples ci-dessous), de guitare et, comme on verra par la suite, même par les chanteurs.

Cahiers d'ethnomusicologie, 10 | 2011 
Ex. 13 : Variations de la cuíca dans l'enregistrement de la samba 0 bem e o mal par Nelson Cavaquinho.

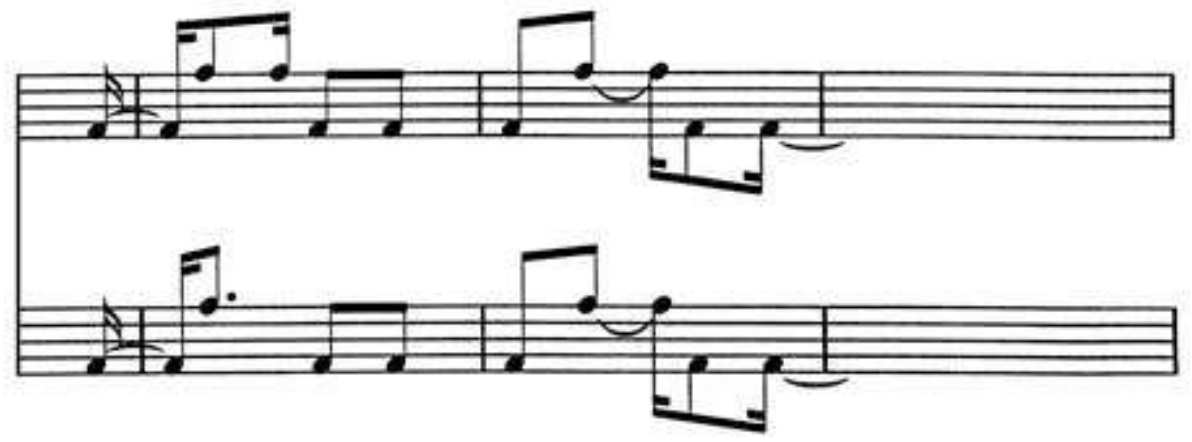

Ex. 14 : Formule jouée par le cavaquinho dans l'enregistrement de la samba Rosa de Ouro par l'ensemble «A voz do morro ».

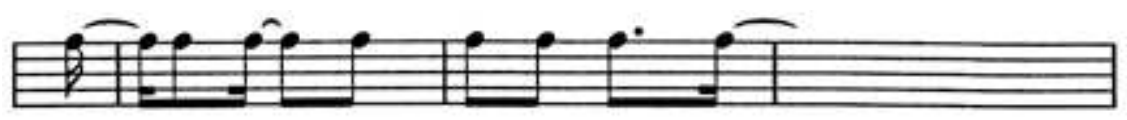

Ex. 15 : Bouteille frappée dans l'enregistrement de la samba Duas horas da manhã par Paulinho da Viola.

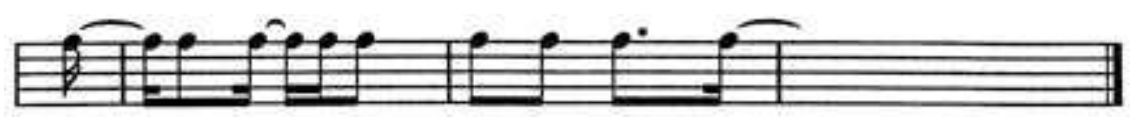

\section{Arranjei um fraseado ${ }^{11}$ : le chant et la batucada}

Si, comme le prétendent la plupart des chercheurs, c'est à l'Estácio qu'eut lieu la création de la samba moderne, il est légitime de supposer que les enregistrements de ce groupe à son époque de gloire devraient présenter des traits des figures rythmiques étudiées cidessus. Or, quand nous avons commencé à écouter les sambas d'Ismael Silva et ses amis dans les enregistrements de la période 1927-1933, nous n'avons pas, dans un premier temps, trouvé de tels traits. Ceci est dû, d'une part, à l'absence d'instruments de la batucada dans la plupart de ces enregistrements, où l'accompagnement était assuré par un orchestre. D'autre part, il est difficile de percevoir, dans ces enregistrements, le cavaquinho, la guitare ou le piano, instruments qui auraient permis d'identifier la modalité rythmique de l'accompagnement (la batida, en jargon carioca). 


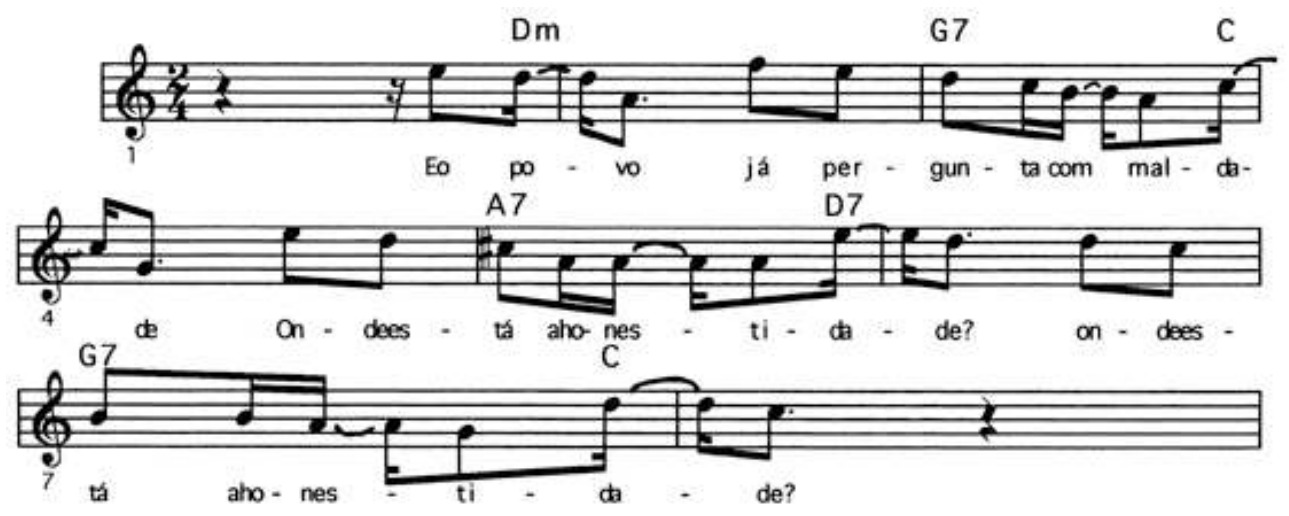

Cependant, ce qui est apparu dans un premier temps comme un handicap s'est transformé en une source de révélations insoupçonnées. En effet, à l'écoute de ces enregistrements, malgré le flou émanant de la section rythmique, la sensation d'écouter une samba de nouveau style était parfaitement réelle. La curiosité aidant, nous avons entrepris de transcrire les mélodies de ces sambas et avons constaté qu'elles étaient construites pour ainsi dire dans le moule rythmique du paradigme de l'Estácio. En d'autres termes, les syllabes de la mélodie étaient articulées, non sur les points préférentiels d'une mesure à $2 / 4$, mais sur ceux prévus par les formules rythmiques que nous avons décrites. Ainsi, le rythme contenu dans les phrases mélodiques suggère celui de la batucada et, autant qu'elle, contribue à caractériser le nouveau style.

Il est connu que les chanteurs populaires influencés par la culture afro-brésilienne ont une forte tendance à chanter en articulant les syllabes (ou une bonne partie d'entre elles) en dehors des points d'appui cométriques prévus par la théorie occidentale de la mesure ${ }^{12}$ . Mais il n'y a, dans la littérature sur la samba, aucune allusion à un "système" d'organisation de cette contramétricité. Brasílio Itiberê affirme même que «ce que l'on rencontre dans le chant populaire [brésilien] est la variété multiple d'une rythmique libre, spontanée, qui sort comme elle sort » (Itiberê 1946 : 115). Or, en étudiant la période 1927-1933, nous avons constaté au contraire, et non sans surprise, l'existence d'un grand nombre de sambas dont les mélodies ont tendance à s'organiser rythmiquement de manière déterminée et non aléatoire. Non seulement elles ont tendance à contrarier la hiérarchie métrique des mesures à $2 / 4$, dans lesquelles elles sont généralement écrites, mais cette contramétricité se produit toujours aux mêmes points d'une période donnée, donc de manière systématique et cyclique.

Cette idée d'une correspondance entre une formule rythmique d'accompagnement et l'articulation rythmique du chant nous a été suggérée tout d'abord par Alejo Carpentier quand, à propos du rythme de claves dans la musique cubaine, il affirma que : «c'est le seul qui peut toujours s'adapter, sans variations, à tous les types de mélodies cubaines, et qui peut donc être considéré comme une sorte de constante scansionnelle » (Carpentier 1979: 55-6) ${ }^{13}$. Ainsi, de la même manière que les mélodies cubaines seraient construites dans le moule du rythme donné par la clave, les mélodies de certaines sambas seraient construites dans celui du rythme du tamborim.

51 La même idée apparaît dans l'ouvrage de Mukuna, qui associe le rythme du tamborim au concept de time line introduit par N'Ketia, et qui serait « un point de référence constant pour la construction de la structure de la phrase d'une chanson, ainsi que pour 
l'organisation métrique linéaire de la phrase». Une telle observation serait, selon Mukuna, pleinement justifiée dans les sambas, où « ce modèle se combine très bien avec les divisions des phrases dans les lignes mélodiques. Pour chaque segment mélodique il y a un cycle rythmique complet » (Mukuna s.d. : 83$)^{14}$. Notre observation a montré que le cycle rythmique s'adapte à la mélodie, non seulement par sa dimension (comme le dit Mukuna), mais aussi par son découpage interne: les articulations rythmiques de la mélodie des sambas en question "tombent " sur les points prévus par la logique de l'imparité rythmique.

Cette affirmation ne se prétend pas valable pour l'ensemble de la samba carioca, d'autant moins pour les sambas pratiquées dans d'autres régions du pays. Notre recherche a été exhaustive seulement en ce qui concerne les enregistrements commerciaux de samba faits à Rio de Janeiro entre 1927 et 1933 par le chanteur Francisco Alves, dont le rôle dans la diffusion des compositions de l'Estácio a été décisif. Dans ce corpus, nous avons constaté que les mélodies dont l'articulation rythmique a tendance à s'identifier au nouveau paradigme sont, et ce n'est pas un hasard, surtout celles d'Ismael Silva et de ses proches (en particulier Noel Rosa).

Pour conclure, nous avons choisi un exemple particulièrement frappant du rapport entre l'articulation rythmique de la mélodie et le paradigme de l'Estácio : le refrain de la samba Onde está a honestidade?, composée par Noel Rosa en 1933. Mais on pourrait en citer beaucoup d'autres, comme A malandragem (Bide-Alves 1928), Se você jurar (Silva-BastosAlves 1931), Nem assim (Gradim 1931) etc. Par ailleurs, une observation non systématique a montré l'existence du même rapport dans beaucoup de sambas bien plus récentes, même s'il n'est pas aussi évident que dans les cas cités. Il se pourrait bien qu'à partir de 1933, la présence croissante de tamborins, cuícas et surdos dans les studios d'enregistrement ait liberé les compositeurs et chanteurs du besoin de faire appel à la mélodie pour réitérer le paradigme de l'Estácio, que nous considérons comme un véritable marqueur d'identité du nouveau style de samba.

\section{BIBLIOGRAPHIE}

\section{Références}

ALVARENGA Oneyda, 1982, [1946] Música popular brasileira. São Paulo : Duas Cidades.

ARAÚJO Samuel, 1992, Acoustic labour in the timing of everyday life. Urbana : University of Illinois

[Thèse de doctorat en ethnomusicologie].

AROM Simha, 1985, Polyphonies et polyrythmies de Centrafrique. Paris : SELAF.

CABRAL Sérgio, 1974, As Escolas de Samba : o quê, como, quem, quando, onde e porquê. Rio de Janeiro : Fontana.

CABRAL Sérgio, 1990, No tempo de Almirante. Rio de Janeiro : Francisco Alves. 
CARPENTIER Alejo, 1979, La música en Cuba. La Havane : Letras Cubanas.

CÁURIO Rita (éd), 1988, Brasil musical. Rio de Janeiro : Art Bureau.

ITIBERÊ Brasílio, 1946, « Ernesto Nazaré na música brasileira ». Boletim latino americano de música, VI/1, 309-21.

JONES A. M., 1959, Studies in African music. London : Oxford University Press.

KOLINSKI Mieczyslaw, 1960, « Review of Studies in African music by A.M. Jones ». The Musical Quarterly, XLVI/1, 105-110.

KOLINSKI Mieczyslaw, 1973, « A cross-cultural approach to rhythmic patterns ». Ethnomusicology, XVII/3, 494-506.

KUBIK Gerhard, 1979, Angolan traits in Black music, games and dances of Brazil. Lisboa : Junta de Investigações Científicas do Ultramar.

MATOS Cláudia, 1982, Acertei no milhar : samba e malandragem no tempo de Getúlio. Rio de Janeiro : Paz e Terra.

MÁXIMO João et DIDIER Carlos, 1990, Noel Rosa. Brasília : UnB.

MOURA Roberto, 1983, Tia Ciata e a pequena África no Rio de Janeiro. Rio de Janeiro : Funarte.

MUKUNA Kazadi-wa, s.d., Contribuição bantu na música popular brasileira. São Paulo : Global.

NKETIA J.H. Kwabena, 1975, The music of Africa. London : Victor Gollancz.

SILVA Marília T. Barboza da, et OLIVEIRA FILHO Arthur L. de., 1989, Cartola. Rio de Janeiro : Funarte.

SOARES Maria Tereza Mello., 1985, São Ismael do Estácio. Rio de Janeiro : Funarte.

TINHORÃO José Ramos, 1990, História social da música popular brasileira. Lisboa : Caminho.

WADDEY Ralph, 1981, « Samba de viola and viola de samba in the Reconcavo of Bahia (Brazil). Part II : samba de viola », Latin American Music Review, 2/2, 252-279.

WATERMAN Richard, 1967, "African Influence on the Music of the Americas », in Sol Tax, ed. : Acculturation in the Americas. New York : Cooper Square : 207-218.

\section{Enregistrements cités}

Pelo telefone (Donga-Mauro de Almeida), chanté par Bahiano, Casa Edison-Odéon 121.322, janvier 1917. Réédition sur CD dans la compilation « Historia del carnaval de Brasil 1902-1950 », vol. I, Ubatuqui Records (Suisse), 1992.

A malandragem (Bide-Francisco Alves), chanté par F. Alves, Odéon 10.113-B, février 1928.

Se você jurar (Ismael Silva-Nilton Bastos-Francisco Alves), chanté par F. Alves et Mário Reis, 1931, rééditée sur CD dans la compilation « Historia del carnaval de Brasil 1902-1950 », vol. I, Ubatuqui Records (Suisse), 1992.

Nem assim (Gradim), chanté par F. Alves et Mário Reis, Odéon 10.824-A, 1931.

"Aquarelas do Brasil », émission de radio produite par Almirante, 4 mai 1945, editée dans la cassette «Escolas de Samba - n 1 », Collector's, Rio de Janeiro, 1996.

Onde a dor não tem razão (Paulinho da Viola-Elton Medeiros), chanté par Paulinho da Viola, LP «Paulinho da Viola », RCA, 1981. 
Rosa de Ouro (Paulinho da Viola-Elton Medeiros-Hermínio Bello de Carvalho), chanté par l'ensemble « A voz do morro », LP « Rosa de Ouro », 1965.

Duas horas da manhã (Nelson Cavaquinho-Ari Monteiro), chanté par Paulinho da Viola, compilation sur CD « Nelson Cavaquinho - Quando eu me chamar saudade », EMI, 1990.

O bem e o mal (Nelson Cavaquinho-Guilherme de Brito), chanté par Nelson Cavaquinho, compilation sur CD « Nelson Cavaquinho - Quando eu me chamar saudade », EMI, 1990.

\section{NOTES}

1. En portugais, le mot «samba » appartient au genre masculin. Nous avons hésité longtemps sur le fait de l'écrire au féminin. Nous aimerions pouvoir dire, en français, « le samba » : cela nous semble plus proche du sens que ce mot a pour les Brésiliens. Mais nous avons considéré que, si le mot «samba » appartient désormais à la langue française aussi, c'est aux francophones et non à nous de décider du genre qu'ils veulent lui attribuer.

2. Les références des enregistrements des sambas citées se trouvent à la fin de l'article.

3. Le passage devint classique, étant cité entre autres par Soares (1985: 94), Matos (1982: 40), Tinhorão (1990 : 232), Moura (1983: 123-4) et (Cáurio $1988: 129)$.

4. Dans les deux exemples suivants, nous avons divisé par deux les valeurs rythmiques employées dans l'ouvrage de Mukuna. Là où nous écrivons « croche, croche, double croche, etc. », il avait donc écrit «noire, noire, croche, etc. ». Nous avons voulu, par ce petit changement, faciliter la comparaison avec les exemples d'autres auteurs cités plus loin.

5. Le lundu est un genre de chanson d'inspiration afro-brésilienne très répandu au Brésil au XIX siècle.

6. "Anyone familiar with Brazilian street samba, as it can be seen at Carnival time in Rio de Janeiro [...] might be conscious of a characteristic percussive pattern wich permeats this music as a most persistant trait. It can be played on various instruments, for instance on a high pitched drum [...], or even on a guitar. It is a focal element in which all other instrumentalists, the singers and dancers find a pivot point for their orientation. "

7. La notation de Kubik ne fait pas de différence entre des «silences" et des "prolongations du son », puisque les deux valeurs y sont exprimées par des points. En effet, cette différence n'est pas pertinente en ce qui concerne les phénomènes rythmiques en question. Nous aurions donc pu transcrire « $\mathrm{x}$. " " par une croche pointée ou par une double croche suivie d'un demi-soupir. Nous avons choisi la première possibilité pour des raisons de simplicité graphique.

8. Nous avons adopté dans cette transcription la convention pratiquée dans la musique populaire brésilienne depuis Pelo telefone, qui fait écrire les sambas en mesure 2/4. Le rythme du tamborim, par conséquent, ne complète son cycle qu'au bout de deux mesures.

9. Sur le programme « Aquarelas do Brasil » voir Cabral (1990: 231).

10. Tous ces rythmes sont cités par Arom (1985: 430), qui les a, lui aussi, trouvés en Centrafrique. En fait, il dresse la liste des possibilités prévues par la formule $2 n=3+(n-2)+3+(n-4)$, sans cependant énoncer cette dernière.

11. C'est le titre d'une samba de Noel Rosa (1933), qui peut approximativement être traduit par : "J'ai trouvé une belle suite de phrases ", de phrases verbales et, par extension, musicales.

12. Voir par exemple Waddey (1981 : 256), et Itiberê (1946: 315). Waterman (1967) discute la même question, du point de vue de la musique afro-américaine en général.

13. "Es el único que puede ajustarse siempre, sin variantes, a todos los tipos de melodías cubanas, constituyendo, por lo tanto, una espécie de constante escansionál ». Carpentier, de son côté, s'inspire du musicologue cubain Emilio Grenet. 
14. Mukuna renvoie à deux exemples musicaux, donnés aux pages 85-87 de son livre, où le cycle rythmique en question est écrit en $16 / 8$, et la mélodie en $2 / 4$. Cela implique qu'un cycle rythmique du tamborim correspond à quatre mesures du chant. Or, notre observation a montré que la proportion est de deux à un, et non de quatre à un, ce qui nous amène à considérer avec réserve ces transcriptions de Mukuna.

\section{RÉSUMÉS}

L'histoire de la samba à Rio de Janeiro peut être divisée en deux styles correspondant à deux périodes successives. Dans le premier (1917-1930 environ), le genre se confondait encore avec le vieux maxixe, dansé dans la ville depuis 1880. Dans le second, créé à partir de 1928 environ dans le quartier de l'Estácio de Sá, la samba contemporaine a trouvé, à quelques retouches près, sa version définitive. L'existence de ces deux styles a été souvent remarquée dans la littérature sur le sujet, mais sans aucune description détaillée de leurs caractéristiques musicales. Or, un groupe de formules rythmiques associées en particulier au tamborim paraît avoir joué un rôle décisif dans la définition du nouveau style. Pour cerner les propriétés de ce groupe, nous avons créé le concept d'un "paradigme de l'Estácio ». Ce paradigme relève de ce que Simha Arom a appelé, dans ses études sur la musique africaine, l'imparité rythmique. L'étude des disques de la période 1927-1933 montre que, avant même que le tamborim soit largement admis dans les studios d'enregistrement, les formules rythmiques en question s'entendaient dans les mélodies des sambas composées à l’Estácio.

The history of the Samba in Rio de Janeiro may be divided into two styles relating to two successive periods. In the first, 1917 to approximately 1930, the genre was still mixed with the old maxixe, which had been danced in the city since 1880. In the second, created in the Estacio de Sa district from roughly 1928 onwards, contemporary Samba found, with one or two minor exceptions, its definitive present day form. The existence of two styles has often been referred to in literature on the subject, but without detailed description of their musical characteristics. It should be noted however, that a group of rhythmic formulas especially associated with the tamborim would seem to have played a decisive role in the definition of the new style. To determine the properties of this group we have created the concept of a "paradigm of the Estacio". This paradigm reveals what Simha Arom, in his studies of African music called rhythmical non parity. Study of records of the 1927 to 1933 period shows that even before the tamborim gained widespread admittance to recording studios, the rhythmical formulas in question were heard in melodies composed in the Estacio.

\section{AUTEUR}

\section{CARLOS SANDRONI}

Carlos SANDRONI est né en 1958 à Rio de Janeiro. Il a suivi des études en sciences sociales dans sa ville natale avant de venir en France pour y passer un doctorat en Musicologie à l'Université de Tours. Guitariste et compositeur, il a vu plusieurs de ses chansons enregistrées par des chanteurs populaires brésiliens. Il a publié Mário contra Macunaíma, un essai sur la culture et la politique 
chez l'écrivain et musicologue brésilien Mário de Andrade. Actuellement il enseigne au Département de Musique de l'Université du Pernambouc (Recife). 